\title{
Relaxation rates of the linearized Uehling-Uhlenbeck equation for bosons
}

\author{
Erich Gust and L. E. Reichl \\ Center for Complex Quantum Systems and Department of Physics, The University of Texas at Austin, Austin, Texas 78712, USA
}

(Received 21 January 2010; revised manuscript received 8 April 2010; published 16 June 2010)

\begin{abstract}
We linearize the Uehling-Uhlenbeck equation for bosonic gases close to thermal equilibrium under the assumption of a contact interaction characterized by a scattering length $a$. We show that the spectrum of relaxation rates is similar to that of a classical hard-sphere gas. However, the relaxation rates show a significant dependence on the fugacity $z$ of the gas, increasing by as much as $60 \%$ of their classical value for $z$ approaching 1 . The relaxation modes are also significantly altered at higher values of $z$. The relaxation rates and modes are determined by the eigenvalues and eigenvectors of a Fredholm integral operator of the second kind. We derive an analytical form for the kernel of this operator and present numerical results for the first few eigenvalues and eigenvectors.
\end{abstract}

DOI: 10.1103/PhysRevE.81.061202

PACS number(s): 51.10.+y, 51.90.+r, 05.30.Jp, 05.60.Gg

\section{INTRODUCTION}

The Uehling-Uhlenbeck (U-U) equation [1], also known as the Boltzmann-Nordheim equation [2], is a semiclassical extension of the Boltzmann equation that accounts for the identity of the particles. As a nonlinear integro-differential equation, it admits a large variety of possible theoretical approaches. In this report, we concern ourselves with its behavior when applied to systems which are spatially uniform and close to thermal equilibrium. These restrictions allow us to linearize the equation about an equilibrium distribution and write it as an eigenvalue equation with a linear integral collision operator.

In a spatially uniform system, the eigenvalues of the collision operator are directly related to the rate of relaxation of the velocity distribution to the equilibrium distribution. In subsequent sections, we provide a full analysis of the linearized U-U equation by computing its relaxation rates and relaxation modes and expressing the relaxation of the fluid in terms of a spectral decomposition involving these rates and modes. We derive analytical formulas as far as possible, and then apply numerical methods to obtain the final results.

In Sec. II, we write the U-U equation in terms of dimensionless quantities and extract a single constant $\gamma$ that gives scale of the relaxation rates for a given type of gas. In Sec. III, we linearize the equation and define the functions that will be used to construct the linear integral operator. We then discuss, in Sec. IV, how the angular dependence is integrated out and how the eigenvalue equation is formed. In Sec. V we discuss our method for converting the integral operator into a finite matrix, and in Sec. VI we present plots of the numerical eigenvalues and eigenvectors. We end in Sec. VII with a discussion of our results and comparisons to the classical cases and other works on the U-U equation.

\section{UEHLING-UHLENBECK EQUATION}

We consider a dilute gas of bosons of mass $m$ in free space which interact pairwise via a contact potential of the form $U(\mathbf{r})=U_{0} \delta^{3}(\mathbf{r})$, where $U_{0}=4 \pi \hbar^{2} a / m$, $\hbar$ is Planck's constant, and $a$ is the $s$-wave scattering length [3]. The kinetic equation that describes relaxation to thermodynamic equilibrium of this boson gas (above the condensation temperature) is the U-U equation which, for a spatially homogeneous gas, can be written as [4]

$$
\begin{aligned}
\frac{d f_{1}(t)}{d t}= & -\frac{U_{0}^{2}}{16 \pi^{5} \hbar} \int d \mathbf{k}_{2} d \mathbf{k}_{3} d \mathbf{k}_{4} \delta\left(\epsilon_{1}+\epsilon_{2}-\epsilon_{3}-\epsilon_{4}\right) \\
& \times \delta^{3}\left(\mathbf{k}_{1}+\mathbf{k}_{2}-\mathbf{k}_{3}-\mathbf{k}_{4}\right)\left\{f_{1}(t) f_{2}(t)\left[1+f_{3}(t)+f_{4}(t)\right]\right. \\
& \left.-\left[1+f_{1}(t)+f_{2}(t)\right] f_{3}(t) f_{4}(t)\right\} .
\end{aligned}
$$

In Eq. (1), $f_{i}(t)=f\left(\mathbf{k}_{i}, t\right)$ is the phase-space density of particles with wave vector $\mathbf{k}_{i}$, and $\epsilon_{i}=\hbar^{2} k_{i}^{2} / 2 m$ is the kinetic energy of a particle with wave vector $\mathbf{k}_{i}$. To complete the statement of the problem, one must specify the distribution function $f(\mathbf{k}, 0)$ at some initial time $t=0$. This initial condition completely determines the distribution at all later times.

The U-U equation has five conserved quantities corresponding to the five conserved quantities in a two-body collision. These quantities are total particle number, total momentum, and total energy. Since the system is spatially uniform, these conserved quantities are given by the particle density $\bar{n}=\int\left[d \mathbf{k} /(2 \pi)^{3}\right] f(\mathbf{k}, t)$, the momentum density $\overline{\mathbf{p}}$ $=\int\left[d \mathbf{k} /(2 \pi)^{3}\right] \hbar \mathbf{k} f(\mathbf{k}, t)$, and the energy density $\bar{\epsilon}$ $=\int\left[d \mathbf{k} /(2 \pi)^{3}\right]\left(\hbar^{2} k^{2} / 2 m\right) f(\mathbf{k}, t)$. The quantities $\bar{n}, \overline{\mathbf{p}}$, and $\bar{\epsilon}$, being conserved, are independent of the time $t$ at which they are evaluated. For convenience, we assume that a reference frame has been chosen such that the momentum density $(\overline{\mathbf{p}})$ of the gas is zero.

The stationary solutions of the U-U equation have the form

$$
f^{0}(\mathbf{k}, z, T)=\frac{z}{e^{\hbar^{2} k^{2} /\left(2 m k_{B} T\right)}-z}
$$

where $k_{B}$ is the Boltzmann constant, $T$ is the temperature, $z$ $=e^{\mu / k_{B} T}$ is the fugacity, and $\mu$ is the chemical potential. The solutions $f(\mathbf{k}, t)$ to Eq. (1) have the property $\lim _{t \rightarrow \infty} f(\mathbf{k}, t)$ $=f^{0}(\mathbf{k}, z, T)$. Since the conserved quantities are independent of time, they can equally well be evaluated using the equilibrium distribution function $f^{0}(\mathbf{k}, z, T)$. Doing this, we find that $\bar{n}$ and $\bar{\epsilon}$ are related to $z$ and $T$ by 


$$
\begin{gathered}
\bar{n}=\frac{1}{\lambda_{T}^{3}} \mathrm{Li}_{3 / 2}(z), \\
\bar{\epsilon}=\frac{3}{2} \frac{k_{B} T}{\lambda_{T}^{3}} \mathrm{Li}_{5 / 2}(z),
\end{gathered}
$$

where

$$
\lambda_{T}=\frac{h}{\sqrt{2 \pi m k_{B} T}}
$$

is the thermal wavelength and $\operatorname{Li}_{s}(z)$ is the polylogarithm function which is defined by

$$
\operatorname{Li}_{s}(z)=\sum_{j=1}^{\infty} \frac{z^{j}}{j^{s}} .
$$

When presented with an arbitrary initial distribution $f(\mathbf{k}, 0)$ one would determine $\bar{n}$ and $\bar{\epsilon}$ and use Eqs. (3) and (4) to find its fugacity and temperature.

We can write the $\mathrm{U}-\mathrm{U}$ equation in dimensionless form if we introduce the dimensionless velocity $\mathbf{c}_{i}=\left(\hbar / \sqrt{2 m k_{B} T}\right) \mathbf{k}_{i}$. In terms of this quantity, the U-U equation (1) takes the form

$$
\begin{aligned}
\frac{d f_{1}(t)}{d t}= & -\frac{\gamma}{\pi^{2} z} \int d \mathbf{c}_{2} d \mathbf{c}_{3} d \mathbf{c}_{4} \delta\left(c_{1}^{2}+c_{2}^{2}-c_{3}^{2}-c_{4}^{2}\right) \\
& \times \delta^{3}\left(\mathbf{c}_{1}+\mathbf{c}_{2}-\mathbf{c}_{3}-\mathbf{c}_{4}\right)\left\{f_{1}(t) f_{2}(t)\left[1+f_{3}(t)+f_{4}(t)\right]\right. \\
& \left.-\left[1+f_{1}(t)+f_{2}(t)\right] f_{3}(t) f_{4}(t)\right\},
\end{aligned}
$$

where the rate constant $\gamma$ is given by

$$
\gamma=\frac{32 \pi z k_{B} T}{h}\left(\frac{a}{\lambda_{T}}\right)^{2} .
$$

A factor of $z$ has been incorporated into $\gamma$ to give it the proper behavior as $z \rightarrow 0$. Consideration of Eq. (3) shows that $\bar{n}=z / \lambda_{T}^{3}$ in the limit that $z \rightarrow 0$. A few algebraic manipulations reveal that $\gamma$ is also equal to $\bar{n} \bar{v} \sigma$, where $\bar{v}$ $=\sqrt{8 k_{B} T / \pi m}$ is the average particle speed and $\sigma=8 \pi a^{2}$ is the total cross section for low-energy scattering of two identical bosons [3]. A nearly identical rate constant is found in the Boltzmann equation with $\sigma=\pi d^{2}$ for classical hard spheres of diameter $d[5]$.

The particular value of $\gamma$ will depend on experimental conditions such as temperature, density, and type of particle. If one knows the detailed properties of the system, the rate constant $\gamma$ can be used to get a rough estimate for the relaxation rate. For example, if we consider sodium atoms in the $F=1, m_{F}=-1$ state [3] at a density of $\bar{n}=10^{11} \mathrm{~cm}^{-3}$ and a temperature of $T=200 \mu \mathrm{K}[6]$, we find that $\gamma \simeq 0.093 \mathrm{~s}^{-1}$. Alternatively, measurement of $\gamma$ by measuring the relaxation rates could provide a way to obtain the scattering length $a$ of the interaction as done in [7].

\section{LINEARIZATION OF THE UEHLING-UHLENBECK EQUATION}

The linearized U-U equation describes the relaxation of the boson gas when it has been perturbed away from ther- modynamic equilibrium, $f^{0}(\mathbf{c})=z /\left(e^{c^{2}}-z\right)$. We choose the linearization scheme,

$$
f(\mathbf{c}, t)=f^{0}(\mathbf{c})+f^{0}(\mathbf{c})\left[1+f^{0}(\mathbf{c})\right] g(\mathbf{c}, t),
$$

where $g(\mathbf{c}, t)$ is a small parameter that approaches zero as the gas equilibrates and thus $f(\mathbf{c}, t)$ approaches $f^{0}(\mathbf{c})$. Applying this scheme to Eq. (7) and keeping only terms linear in $g(\mathbf{c}, t)$, we obtain the linearized U-U equation,

$$
\begin{aligned}
\frac{d g_{1}(t)}{d t}= & -\frac{\gamma}{\pi^{2} z} \frac{1}{1+f_{1}^{0}} \int d \mathbf{c}_{2} d \mathbf{c}_{3} d \mathbf{c}_{4} \delta^{3}\left(\mathbf{c}_{1}+\mathbf{c}_{2}-\mathbf{c}_{3}-\mathbf{c}_{4}\right) \\
& \times \delta\left(c_{1}^{2}+c_{2}^{2}-c_{3}^{2}-c_{4}^{2}\right) f_{2}^{0}\left(1+f_{3}^{0}\right)\left(1+f_{4}^{0}\right) \\
& \times\left[g_{1}(t)+g_{2}(t)-g_{3}(t)-g_{4}(t)\right],
\end{aligned}
$$

where $f_{i}^{0}=f^{0}\left(\mathbf{c}_{i}\right)$ and $g_{i}(t)=g\left(\mathbf{c}_{i}, t\right)$. In all expressions, the classical (Boltzmann) limit can be obtained by considering $z \rightarrow 0$. In this limit, $f^{0}(\mathbf{c}) \rightarrow z e^{-c^{2}}$ and $1+f^{0}(\mathbf{c}) \rightarrow 1$. We have also used the fact that $\left(1+f_{1}^{0}\right)\left(1+f_{2}^{0}\right) f_{3}^{0} f_{4}^{0}=f_{1}^{0} f_{2}^{0}\left(1+f_{3}^{0}\right)\left(1+f_{4}^{0}\right)$ to write Eq. (10) in a form where its classical limit is clearly the linearized Boltzmann equation [8].

We can see by inspection that Eq. (10) admits five forms of $g(\mathbf{c}, t)$ which result in $\frac{d g(\mathbf{c}, t)}{d t}=0$. These are $g(\mathbf{c}, t) \propto 1$, $g(\mathbf{c}, t) \propto c^{2}$, and $g(\mathbf{c}, t)$ proportional to any of the three components of c. These five functions represent the five conserved quantities of the U-U equation. In Sec. IV, we will see that each of these forms corresponds to an eigenfunction with zero eigenvalue. We will also use knowledge of these forms to obtain analytical expressions for the first two eigenfunctions.

Since the right side of Eq. (10) is linear in $g(\mathbf{c}, t)$, it can be written as a convolution of the function $g(\mathbf{c}, t)$. Doing this, we find that

$$
\begin{aligned}
\frac{d g_{1}(t)}{d t}= & -\gamma M\left(\mathbf{c}_{1}\right) g_{1}(t)-\frac{\gamma}{2 \pi z} \frac{1}{1+f_{1}^{0}} \\
& \times\left[\int d \mathbf{c}_{2} f_{2}^{0} Q\left(\mathbf{c}_{1}, \mathbf{c}_{2}\right) g_{2}(t)-2 \int d \mathbf{c}_{3} f_{3}^{0} S\left(\mathbf{c}_{1}, \mathbf{c}_{3}\right) g_{3}(t)\right],
\end{aligned}
$$

where the kernels $Q$ and $S$ are given by

$$
\begin{aligned}
Q\left(\mathbf{c}_{1}, \mathbf{c}_{2}\right) \equiv & \frac{2}{\pi} \int d \mathbf{c}_{3} d \mathbf{c}_{4} \delta^{3}\left(\mathbf{c}_{1}+\mathbf{c}_{2}-\mathbf{c}_{3}-\mathbf{c}_{4}\right) \\
& \times \delta\left(c_{1}^{2}+c_{2}^{2}-c_{3}^{2}-c_{4}^{2}\right)\left(1+f_{3}^{0}\right)\left(1+f_{4}^{0}\right), \\
S\left(\mathbf{c}_{1}, \mathbf{c}_{3}\right) \equiv & \frac{1+f_{3}^{0}}{f_{3}^{0}} \frac{2}{\pi} \int d \mathbf{c}_{2} d \mathbf{c}_{4} \delta^{3}\left(\mathbf{c}_{1}+\mathbf{c}_{2}-\mathbf{c}_{3}-\mathbf{c}_{4}\right) \\
& \times \delta\left(c_{1}^{2}+c_{2}^{2}-c_{3}^{2}-c_{4}^{2}\right) f_{2}^{0}\left(1+f_{4}^{0}\right),
\end{aligned}
$$

and the function $M$ is given by

$$
M\left(\mathbf{c}_{1}\right)=\frac{1}{2 \pi z\left(1+f_{1}^{0}\right)} \int d \mathbf{c}_{2} f_{2}^{0} Q\left(\mathbf{c}_{1}, \mathbf{c}_{2}\right) .
$$

The particular form of the kernels $Q$ and $S$ have been chosen so that they are symmetric in their two arguments and reduce 
to their classical expressions in the limit $z \rightarrow 0$. It is possible to write these functions in closed form as

$$
\begin{aligned}
Q\left(\mathbf{c}_{1}, \mathbf{c}_{2}\right)= & \frac{e^{c_{1}^{2}+c_{2}^{2}}}{e^{c_{1}^{2}+c_{2}^{2}}-z^{2}} \\
& \times\left[\frac{2}{\left|\mathbf{c}_{1}+\mathbf{c}_{2}\right|} \ln \left(\frac{e^{c_{1}^{2} / 2+c_{2}^{2} / 2+\left(\left|\mathbf{c}_{1}+\mathbf{c}_{2}\right|\left|\mathbf{c}_{1}-\mathbf{c}_{2}\right|\right) / 2}-z}{e^{c_{1}^{2} / 2+c_{2}^{2} / 2-\left(\left|\mathbf{c}_{1}+\mathbf{c}_{2}\right|\left|\mathbf{c}_{1}-\mathbf{c}_{2}\right|\right) / 2}-z}\right)\right. \\
- & \left.\left|\mathbf{c}_{1}-\mathbf{c}_{2}\right|\right], \\
S\left(\mathbf{c}_{1}, \mathbf{c}_{2}\right)= & \frac{1}{z\left|\mathbf{c}_{1}-\mathbf{c}_{2}\right|\left(e^{-c_{1}^{2}}-e^{-c_{2}^{2}}\right)} \\
& \quad \times \ln \left(\frac{e^{-\left|\mathbf{c}_{1} \times \mathbf{c}_{2}\right|^{2} /\left(\mathbf{c}_{1}-\mathbf{c}_{2}\right)^{2}}-z e^{-c_{2}^{2}}}{e^{-\left|\mathbf{c}_{1} \times \mathbf{c}_{2}\right|^{2} /\left(\mathbf{c}_{1}-\mathbf{c}_{2}\right)^{2}}-z e^{-c_{1}^{2}}}\right) .
\end{aligned}
$$

The details of this derivation are outlined in Appendix A.

\section{EIGENVALUE EQUATION}

The analogy between convolutions and matrix products identifies Eq. (11) as an eigenvalue problem and the eigenvalues determine how quickly the velocity distribution of the gas relaxes to thermal equilibrium. The properties of the kernel determine the form of the eigenvalue spectrum.

Since the linearized Boltzmann equation for hard spheres is a limiting case of Eq. (11), we expect that the spectrum of the linearized U-U equation will have the same features as the spectrum of the linearized Boltzmann equation $[9,10]$. Specifically, the first five eigenvalues of the linearized Boltzmann equation are zero, representing the five conserved quantities. The subsequent nonzero discrete eigenvalues increase but are bounded from above by a limiting value. Beyond this limiting value there is a continuum of eigenvalues with singular eigenfunctions. We expect to find five zero eigenvalues for the linearized U-U equation as well as a sequence of increasing eigenvalues that converge to a limiting value.

\section{A. Angular dependence}

For the linearized Boltzmann equation, the nonzero eigenvalues must be determined numerically $[8,11]$ and at the same is true for the U-U equation. However, we do not need to solve the entire problem numerically. In this section, we convert Eq. (11) into a one-dimensional eigenvalue equation by treating the angular dependence of $g(\mathbf{c}, t)$ analytically.

If we inspect the form of Eqs. (15) and (16), we see that the functions $Q$ and $S$ can be written so that they only depend on the magnitudes of $\mathbf{c}_{1}$ and $\mathbf{c}_{2}$ and the angle $\theta$ between them. Similarly, Eq. (14) only depends on the magnitude of $\mathbf{c}_{1}$. These symmetries result in a decoupling of perturbations with different angular dependences. We begin by expanding the angular dependence of the function $g(\mathbf{c})$ in terms of spherical harmonics $Y_{l}^{m}(\hat{\mathbf{c}})$,

$$
g(\mathbf{c}, t)=\sum_{l=0}^{\infty} \sum_{m=-l}^{l} g_{l, m}(c, t) Y_{l}^{m}(\hat{\mathbf{c}}) .
$$

We also express the functions $Q$ and $S$ as expansions in terms of spherical harmonics. For example,

$$
\begin{aligned}
Q\left(\mathbf{c}_{1}, \mathbf{c}_{2}\right) & \equiv \sum_{l^{\prime \prime}} \frac{2 l^{\prime \prime}+1}{2} Q^{l^{\prime \prime}}\left(c_{1}, c_{2}\right) P_{l^{\prime \prime}}(\cos \theta) \\
& =2 \pi \sum_{l^{\prime \prime}} Q^{l^{\prime \prime}}\left(c_{1}, c_{2}\right) \sum_{m^{\prime \prime}} Y_{l^{\prime \prime}}^{m^{\prime \prime} *}\left(\hat{\mathbf{c}}_{2}\right) Y_{l^{\prime \prime}}^{m^{\prime \prime}}\left(\hat{\mathbf{c}}_{1}\right),
\end{aligned}
$$

where $P_{l}(\cos \theta)$ is a Legendre polynomial and the spherical harmonic addition theorem has been used to obtain the last equality. Equation (18) defines the function $Q^{l}\left(c_{1}, c_{2}\right)$. Written explicitly, it is

$$
Q^{l}\left(c_{1}, c_{2}\right)=\int_{-1}^{1} d(\cos \theta) Q\left(\mathbf{c}_{1}, \mathbf{c}_{2}\right) P_{l}(\cos \theta) .
$$

We obtain similar equations for $S\left(\mathbf{c}_{1}, \mathbf{c}_{2}\right)$ if we replace $Q^{l^{\prime \prime}}\left(c_{1}, c_{2}\right)$ with $S^{l^{\prime \prime}}\left(c_{1}, c_{2}\right)$ in Eqs. (18) and (19).

The function $M\left(\mathbf{c}_{1}\right)$ is defined in terms of $Q\left(\mathbf{c}_{1}, \mathbf{c}_{2}\right)$ in Eq. (14). By using Eq. (18) it simplifies to the form

$$
M\left(c_{1}\right)=\frac{1}{z} \frac{1}{1+f_{1}^{0}} \int_{0}^{\infty} d c_{2} c_{2}^{2} f_{2}^{0} Q^{0}\left(c_{1}, c_{2}\right) .
$$

When expansions (17) and (18) are substituted into Eq. (11), the orthogonality of the spherical harmonics cancels all summations. We are left with the one-dimensional equation

$$
\begin{aligned}
\frac{d g_{l, m}\left(c_{1}, t\right)}{d t}= & -\gamma M\left(c_{1}\right) g_{l, m}\left(c_{1}, t\right) \\
& -\frac{\gamma}{z} \frac{1}{1+f_{1}^{0}} \int d c_{2} c_{2}^{2} f_{2}^{0}\left[Q^{l}\left(c_{1}, c_{2}\right)\right. \\
& \left.-2 S^{l}\left(c_{1}, c_{2}\right)\right] g_{l, m}\left(c_{2}, t\right),
\end{aligned}
$$

in which the entire angular dependence has been reduced to two indices $l$ and $m$. Notice that since $m$ only appears as an index of $g$, the eigenvalues of this equation will not depend on $m$ and will be $(2 l+1)$-fold degenerate for a given value of $l$. The only effect of $l$ is to change the kernel of this equation.

\section{B. Symmetrized eigenvalue equation}

In Eq. (21) we have a complete description of the evolution of the deviations $g_{l, m}(c, t)$ from equilibrium for the case when $g_{l, m}(c, t) \ll 1$. We note that it contains two terms: one that multiplies $g_{l, m}\left(c_{1}, t\right)$ by a function of $c_{1}$ and another that convolves $g_{l, m}\left(c_{2}, t\right)$ with a kernel function of $c_{1}$ and $c_{2}$. The fact that this kernel function is not symmetric under interchange of $c_{1}$ and $c_{2}$ is undesirable when a numerical solution is attempted because a matrix corresponding to this kernel will not be symmetric. To restore this symmetry, we define a new function $h_{l, m}(c, t)$ which will represent the deviations from equilibrium. The function $h_{l, m}(c, t)$ is related to $g_{l, m}(c, t)$ by 


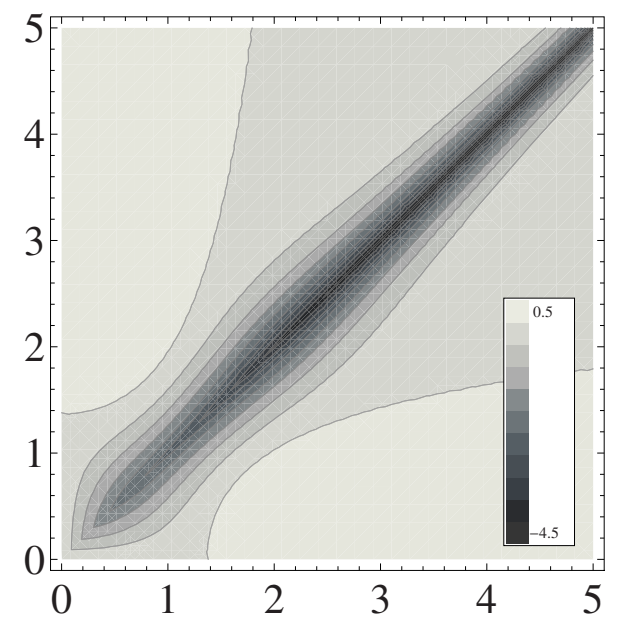

FIG. 1. (Color online) Plot of the function $K^{0}\left(c_{1}, c_{2}\right)$ versus $c_{1}$ and $c_{2}$. The function has a cusp when $c_{1}=c_{2}$, but is continuous over its whole domain. In this plot $z=0.2$.

$$
h_{l, m}(c, t)=c \sqrt{f^{0}(c)\left[1+f^{0}(c)\right]} g_{l, m}(c, t) .
$$

Rewritten in terms of $h_{l, m}(c, t)$ and noting that $f^{0}(c) /$ $\left[1+f^{0}(c)\right]=z e^{-c^{2}}$, Eq. (21) becomes

$$
\begin{aligned}
\frac{d h_{l, m}\left(c_{1}, t\right)}{d t}= & -\gamma\left[M\left(c_{1}\right) h_{l, m}\left(c_{1}, t\right)\right. \\
& \left.+\int d c_{2} K^{l}\left(c_{1}, c_{2}\right) h_{l, m}\left(c_{2}, t\right)\right],
\end{aligned}
$$

where we have defined the symmetric kernel $K^{l}\left(c_{1}, c_{2}\right)$ as

$$
K^{l}\left(c_{1}, c_{2}\right)=c_{1} c_{2} e^{-c_{1}^{2} / 2-c_{2}^{2} / 2}\left[Q^{l}\left(c_{1}, c_{2}\right)-2 S^{l}\left(c_{1}, c_{2}\right)\right] .
$$

Equation (23) is a symmetric Fredholm integral equation of the second kind [12] for the function $h_{l, m}\left(c_{1}, t\right)$. The functions $K^{l}\left(c_{1}, c_{2}\right)$ and $M\left(c_{1}\right)$ appear to be continuous as shown in Figs. 1 and 2. In the Boltzmann limit, $K^{l}\left(c_{1}, c_{2}\right)$ is completely continuous and therefore has a countable set of real eigenvalues. Since $K^{l}\left(c_{1}, c_{2}\right)$ and $M\left(c_{1}\right)$, for nonzero $z$, are computed numerically we will not try to prove the existence of eigenvalues. Nevertheless, we expect Eq. (23) to have a countable set of real eigenvalues due to its similarity with the Boltzmann equation. Let us define an eigenvalue $\lambda_{l}^{n}$ and an eigenfunction $\phi_{l}^{n}(c)$ as the pair which satisfies

$$
\lambda_{l}^{n} \phi_{l}^{n}\left(c_{1}\right)=M\left(c_{1}\right) \phi_{l}^{n}\left(c_{1}\right)+\int d c_{2} K^{l}\left(c_{1}, c_{2}\right) \phi_{l}^{n}\left(c_{2}\right)
$$

These eigenfunctions have an orthonormality condition given by

$$
\int_{0}^{\infty} d c \phi_{l}^{n}(c) \phi_{l}^{n^{\prime}}(c)=\delta_{n, n^{\prime}}
$$

The eigenvalues defined by Eq. (25) are positive and dimensionless. To obtain the relaxation rates in $\mathrm{s}^{-1}$, one must first find $z$ from Eq. (3), which depends on the density and temperature of the gas, and then find $\gamma$ from Eq. (8). Once the

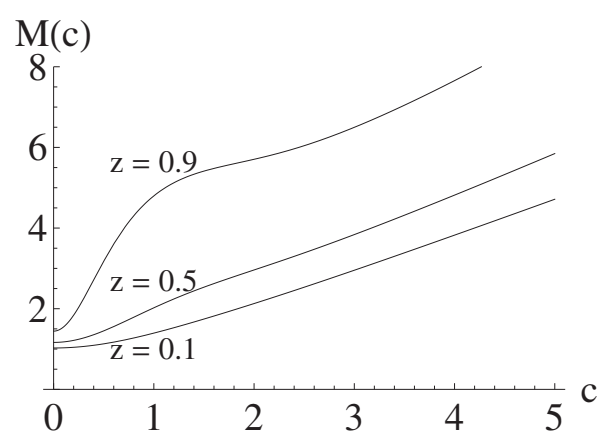

FIG. 2. Plot of the function $M(c)$ versus $c$ for three values of $z$. The function is continuous for all positive values of $c$.

value of $\gamma$ is known in $\mathrm{s}^{-1}$, the relaxation rate of any mode $\phi_{l}^{n}$ is given by $\gamma \lambda_{l}^{n}$.

\section{Eigenfunction expansion of $f(\mathrm{c}, t)$}

From our knowledge of the conserved quantities of the $\mathrm{U}-\mathrm{U}$ equation, the eigenfunction $\phi_{0}^{0}(c)$ should produce a deviation $g(c)$ that is constant. In addition, the eigenfunction $\phi_{1}^{0}(c)$ should produce a deviation proportional to $c$ while the eigenfunction $\phi_{0}^{1}(c)$ should produce a deviation that is proportional to $c^{2}+B$, where $B$ is a constant. The normalization constants of the eigenfunction and the constant $B$ are determined by the condition in Eq. (26). Using this condition, we find that the eigenfunctions are explicitly given by

$$
\begin{gathered}
\phi_{0}^{0}(c)=\sqrt{\frac{4 z}{\sqrt{\pi} \mathrm{Li}_{1 / 2}(z)} \frac{c e^{c^{2} / 2}}{c^{c^{2}}-z},} \\
\phi_{1}^{0}(c)=\sqrt{\frac{8 z}{3 \sqrt{\pi} \mathrm{Li}_{3 / 2}(z)} \frac{c^{2} e^{c^{2 / 2}}}{e^{c^{2}}-z},} \\
\phi_{0}^{1}(c)=\sqrt{\frac{16 z \mathrm{Li}_{1 / 2}(z)}{15 \sqrt{\pi} \mathrm{Li}_{5 / 2}(z) \mathrm{Li}_{1 / 2}(z)-9 \sqrt{\pi} \mathrm{Li}_{3 / 2}^{2}(z)}} \\
\times\left(c^{2}-\frac{3 \mathrm{Li}_{3 / 2}(z)}{2 \operatorname{Li}_{1 / 2}(z)}\right) \frac{c e^{c^{2} / 2}}{e^{c^{2}}-z} .
\end{gathered}
$$

If we now expand the function $h_{l, m}(c, t)$ in terms of the eigenfunctions $\phi_{l}^{n}(c)$ according to

$$
h_{l, m}(c, t)=\sqrt{\frac{4}{\sqrt{\pi} \operatorname{Li}_{1 / 2}(z)}} \sum_{n=0}^{\infty} A_{l, m}^{n} e^{-\lambda_{l}^{n} \gamma t} \phi_{l}^{n}(c),
$$

we find that we can use this along with Eqs. (17) and (22) to write the original distribution $f(\mathbf{c})$ as

$$
f(\mathbf{c}, t)=f^{0}(\mathbf{c})+\frac{1}{c^{2}} \sum_{n=0}^{\infty} \sum_{l=0}^{\infty} \sum_{m=-l}^{l} A_{l, m}^{n} e^{-\lambda_{l}^{n} \gamma t} \phi_{0}^{0}(c) \phi_{l}^{n}(c) Y_{l}^{m}(\hat{\mathbf{c}}) .
$$

The coefficients of expansion $A_{l, m}^{n}$ depend on the initial distribution $f(\mathbf{c}, 0)$ and are given by 


$$
A_{l, m}^{n}=\int d \mathbf{c}\left[f(\mathbf{c}, 0)-f^{0}(c)\right] \frac{\phi_{l}^{n}(c)}{\phi_{0}^{0}(c)} Y_{l}^{m *}(\hat{\mathbf{c}}) .
$$

The entire time dependence of Eq. (31) is carried by the term $e^{-\lambda_{l}^{n} \gamma t}$. Using the forms for $\phi_{l}^{n}(c)$ in Eqs. (27)-(29) one can quickly see that $A_{0,0}^{0}, A_{0,0}^{1}$, and $A_{1, m}^{0}$ are identically zero due to the conservation laws. This ensures that even though the eigenvalues $\lambda_{0}^{0}, \lambda_{0}^{1}$, and $\lambda_{1}^{0}$ are zero, the distribution $f(\mathbf{c}, t)$ decays to the equilibrium state $f^{0}(\mathbf{c})$. We note that an approximation similar to that of Ref. [13] can be obtained by assuming that all modes relax at the same rate.

Equations (31) and (32) allow us to determine the evolution of any distribution function $f(\mathbf{c}, t)$ as long as it is close to the equilibrium distribution $f^{0}(\mathbf{c})$. As mentioned in Sec. IV, we expect that all of the discrete eigenvalues will fall between zero and a finite limiting value, which we denote as $\lambda_{L}(z)$. In Appendix B, we show that

$$
\lambda_{L}(z)=\frac{1}{z} \mathrm{Li}_{2}(z) .
$$

This expression is very useful because it sets the maximum rate of relaxation for any possible perturbation to the velocity distribution of the gas. It includes the Boltzmann limit, $\lambda_{L}(z)=1$ as well as the interesting limit $\lambda_{L}(1)=\pi^{2} / 6$. This finite value of $\lambda_{L}$ for $z=1$ implies that all discrete eigenvalues remain finite. It is well known that a Bose gas undergoes a phase transition to a Bose-Einstein condensate when $z=1$. The absence of any interesting behavior in the eigenvalue spectrum for $z=1$ indicates that the linearized U-U equation is inadequate for describing this phase transition and is of most interest for $0<z<1$.

\section{FINITE ORDINATE METHOD}

We now turn toward the numerical computation of the eigenfunctions $\phi_{l}^{n}(c)$ which have nonzero eigenvalues $\lambda_{l}^{n}$. In order to compute the eigenfunctions and eigenvalues of Eq. (25) we discretize the eigenvalue equation and choose an appropriate truncation size for the resulting matrix.

There are several ways to discretize an equation like Eq. (25); one of which is expansion in terms of a set of orthogonal polynomials. Most sets of orthogonal polynomials are ill suited as bases for these types of problems. The standard expansion in Laguerre polynomials suffers from very slow convergence because these polynomials are proportional to $c^{2}$ for $c \rightarrow 0$ while the true eigenfunctions are proportional to $c$ [11]. While it is possible to choose a more appropriate set of orthogonal polynomials, they all share the same disadvantage. One must compute the matrix element of the kernel in the polynomial basis and this is often a lengthy multidimensional quadrature calculation. Finite element methods offer another option, but these also require evaluation of the same types of integrals of the kernel, albeit with simpler basis functions.

Ultimately, we want to avoid as many integrations as possible so we choose a finite ordinate method based on the work of Shizgal [11]. In this method, a quadrature scheme for evaluating integrals on $[0, \infty)$ is chosen, and the integral in Eq. (25) is evaluated directly with this scheme. We denote the evaluation points as $x_{i}$, the evaluation weights as $w_{i}$, and the total number of quadrature points as $N_{Q}$. If we then demand that Eq. (25) be satisfied for $c_{1}$ equal to any evaluation point $x_{j}$, we obtain a standard matrix eigenvalue equation,

$$
\lambda_{l}^{n} \phi_{l}^{n}\left(x_{i}\right)=M\left(x_{i}\right) \phi_{l}^{n}\left(x_{i}\right)+\sum_{j=1}^{N_{Q}} w_{j} K^{l}\left(x_{i}, x_{j}\right) \phi_{l}^{n}\left(x_{j}\right) .
$$

To simplify the notation we will write $\phi_{i}=\phi_{l}^{n}\left(x_{i}\right), M_{i}$ $=M\left(x_{i}\right)$, and $K^{l}\left(x_{i}, x_{j}\right)=K_{i j}^{l}$. We leave the dependence on $l$ and $n$ as implied for the remainder of this section. Notice that our attempt to produce a symmetric matrix has potentially been foiled by the appearance of the quadrature weights $w_{i}$. This problem could be solved by only using quadrature schemes with equal $w_{i}$, but this would mean limiting ourselves to the midpoint or trapezoid methods. This limitation severely decreases the convergence rate of the eigenvalue as $N_{Q}$ is increased. Therefore, we choose to solve the problem instead by defining

$$
\bar{\phi}_{i}=\sqrt{w_{i}} \phi_{i}
$$

The eigenvalue equation in terms of $\bar{\phi}_{i}$ is now

$$
\lambda \bar{\phi}_{i}=M_{i} \bar{\phi}_{i}+\sum_{j=1}^{N_{Q}} \sqrt{w_{i} w_{j}} K_{i j}^{l} \bar{\phi}_{j} .
$$

This equation allows us to use any quadrature scheme for $x_{i}$ and $w_{i}$. We would like to point out that the quantity $\bar{\phi}_{i}$ is purely a computational tool and holds no physical meaning. It is the quantity $\phi_{i}$ that represents an eigenfunction of Eq. (25).

We can use the same quadrature scheme to compute the values $M_{i}$ by evaluating the integral in Eq. (20). The result is

$$
M_{i}=\left(1-z e^{-x_{i}^{2}}\right) \sum_{j=1}^{N_{Q}} w_{j} x_{j}^{2} \frac{1}{e^{x_{j}^{2}}-z} Q^{0}\left(x_{i}, x_{j}\right) .
$$

After numerically computing the array of values $K_{i j}^{l}$ and $M_{i}$ we define a symmetric matrix $\mathbf{H}^{l}$ as

$$
H_{i j}^{l}=M_{i} \delta_{i j}+\sqrt{w_{i} w_{j}} K_{i j}^{l} .
$$

In the next section, we describe how we find the eigenvalues and eigenvectors of $\mathbf{H}^{l}$ numerically.

\section{NUMERICAL RESULTS FOR EIGENVALUES AND EIGENVECTORS}

To compute the eigenvalues and eigenvectors, we begin by choosing a quadrature scheme as outlined in Sec. V. This gives us a set of evaluation points $x_{i}$ and quadrature weights $w_{i}$. In general, it is not optimal to choose equally spaced evaluation points due to the observed rapid oscillations of the higher-order eigenfunctions near the origin. The issue becomes more pronounced for higher values of $z$.

For our quadrature scheme, we chose a fixed cutoff value of $c=6$. This effectively assumes that the velocity perturbations are zero beyond $c=6$. Plots of the eigenfunctions con- 


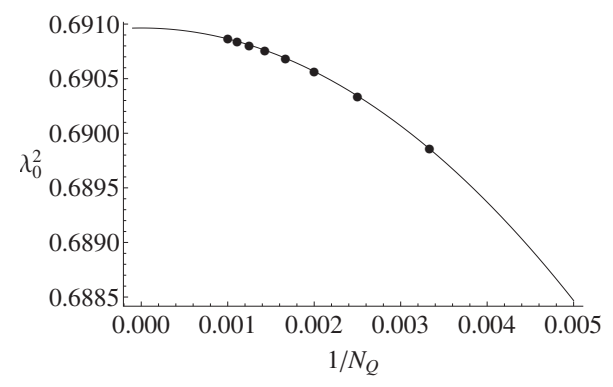

FIG. 3. Plot of the first nonzero eigenvalue versus inverse matrix size which illustrates the fitting procedure used to estimate the exact eigenvalues. In this plot $z=0.1$.

firm this assumption. We then subdivide the interval $0<c$ $<6$ into $N_{Q} / 5$ subintervals, with the width of each subinterval proportional to $c^{p}$, with $p=3$ in the final data. In this way we get a much higher density of subintervals near $c=0$. We then apply a five-point Gaussian quadrature to each subinterval. The use of a five-point method on $N_{Q} / 5$ subintervals results in a total of $N_{Q}$ evaluation points. This scheme was repeated with differing values $p$ and increasing values of $N_{Q}$ to gauge its accuracy. We found that schemes with $p=3$ produced accurate results without having to resort to large values of $N_{Q}$.

In the second step, we choose a particular value of $l$ and $z$ and use a Gauss-Kronrod local adaptive quadrature scheme $(G 7, K 15)[14]$ to evaluate the integral in Eq. (19) at the evaluation points $x_{i}$. Note that this quadrature is used to evaluate the integral over $\alpha$ and is distinctly different than the scheme for integration over $c_{2}$ mentioned above. We use this in combination with Eqs. (15) and (16) to obtain the $N_{Q} \times N_{Q}$ matrices $Q_{i j}^{0}, Q_{i j}^{l}$, and $S_{i j}^{l}$. From these we compute the values $M_{i}$ according to Eq. (37) and the matrices $\mathbf{K}^{l}$ and $\mathbf{H}^{l}$ according to Eqs. (24) and (38). We then numerically diagonalize the matrix $\mathbf{H}^{l}$ for increasing $N_{Q}$ and find its eigenvalues and eigenvectors.

To obtain an accurate estimate of the exact eigenvalues, we do not use the numerical eigenvalues of any single matrix. Instead, we plot the numerical eigenvalues versus the inverse matrix size $1 / N_{Q}$. We then fit this plot with a polynomial in $1 / N_{Q}$ to extrapolate the value of intercept with the vertical axis [15]. This value is what we use for our best estimate of the exact eigenvalues. This procedure is illustrated in Fig. 3 and several eigenvalues are reported in Table I. Our procedure was also able to reproduce the classical results for very small values of $z$.

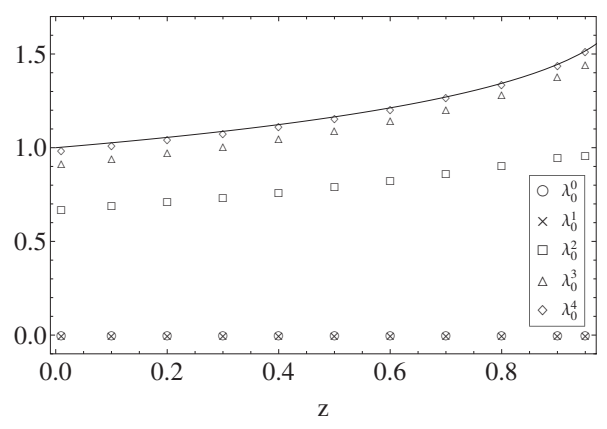

FIG. 4. Plot showing the dependence of the first few $l=0$ eigenvalues on $z$. Note the two zero eigenvalues. The solid curve represents $\lambda_{L}(z)$, the start of the continuous spectrum.

A plot of interest is the dependence of the eigenvalues on $z$. In the classical limit, $z \rightarrow 0$ we expect our eigenvalues to converge to the known classical eigenvalues [8]. We expect that there are two zero eigenvalues when $l=0$ representing conservation of particle number and energy and one zero eigenvalue when $l=1$ representing conservation of momentum. We also expect that all discrete eigenvalues will be between zero and $\lambda_{L}(z)$ as discussed in Sec. IV and Appendix B. The plots of $\lambda$ versus $z$ in Figs. 4 and 5 confirm our expectations. These plots also strongly suggest that the discrete eigenvalues approach finite limiting values between zero and $\pi^{2} / 6$ as $z \rightarrow 1$. As $z$ approaches 1 , the accuracy of the computation decreases substantially. This can be understood by looking at the plots of the eigenfunctions for high values of $z$. Plots of the eigenvectors $\phi_{l}^{n}$ for $z=0.1$ and $z$ $=0.9$ are shown in Figs. 6 and 7. The eigenvectors appear to be continuous and bounded, but they show increasingly rapid variations near the origin as $z$ is increased. As $z$ becomes greater than about 0.95 , the oscillatory features of the higherorder eigenfunctions become smaller than the size of our subintervals and the accuracy is lost.

\section{CONCLUSION}

We have linearized the Uehling-Uhlenbeck equation for a Bose gas interaction with a contact potential and derived explicit forms for the kernels $Q$ and $S$ as functions of the fugacity $z$. We then compute the spectrum of eigenvalues and their eigenfunction. The eigenvalue spectrum has the same form as it does for the linearized Boltzmann equation and the eigenfunctions appear to be continuous and bounded for 0 $\leq z<1$.

TABLE I. Several discrete eigenvalues of the Uehling-Uhlenbeck equation. The uncertainties of all values are less than $10^{-5}$.

\begin{tabular}{lllccccl}
\hline \hline & $\lambda_{0}^{0}$ & $\lambda_{0}^{1}$ & \multicolumn{1}{c}{$\lambda_{0}^{2}$} & $\lambda_{0}^{3}$ & $\lambda_{1}^{0}$ & $\lambda_{1}^{1}$ & $\lambda_{L}(z)$ \\
\hline Classical & 0 & 0 & 0.67123 & 0.91157 & 0 & 0.69503 & 1 \\
$z=0.1$ & 0.0 & 0.0 & 0.69096 & 0.94117 & 0.0 & 0.72104 & 1.02618 \\
$z=0.2$ & 0.0 & 0.0 & 0.71248 & 0.97354 & 0.0 & 0.74994 & 1.05502 \\
$z=0.5$ & 0.0 & 0.0 & 0.79129 & 1.09410 & 0.0 & 0.86163 & 1.16448 \\
$z=0.9$ & 0.0 & 0.0 & 0.94759 & 1.44144 & 0.0 & 1.14435 & 1.44413 \\
\hline \hline
\end{tabular}




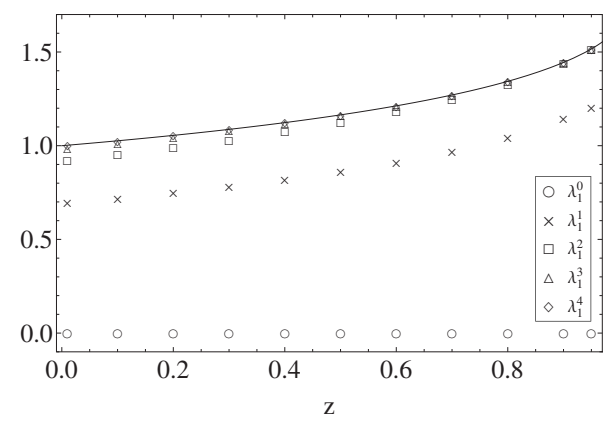

FIG. 5. Plot showing the dependence of the first few $l=1$ eigenvalues on $z$. Note the single zero eigenvalue. The solid curve represents $\lambda_{L}(z)$, the start of the continuous spectrum.

In a spatially uniform system, velocity perturbations corresponding to the eigenfunction $\phi_{l}^{n}$ relax at a rate of $\gamma \lambda_{l}^{n}$, where $\lambda_{l}^{n}$ is the associated eigenvalue and $\gamma$ is defined by Eq. (8). In a nonuniform system, these eigenfunctions can still serve as a basis for expansion of the system's velocity distribution.

The eigenvalues (and therefore the relaxation rates) increase as $z$ increases, but they remain bounded and also remain within an order of magnitude of their classical values. This increase in relaxation rates is due to the Bose enhancement factors of $1+f_{i}(t)$ in the U-U equation which increases the scattering rate compared to the classical Boltzmann equation.

A second interesting result is that the eigenvalues of the linearized U-U equation appear to remain finite in the limit $z \rightarrow 1$. The discrete eigenvalues of the linearized U-U equation all lie between zero and a finite limiting value $\lambda_{L}(z)$ $=\frac{1}{z} \mathrm{Li}_{2}(z)$. Since a Bose gas undergoes a phase transition when $z=1$, we would expect a more interesting behavior of the eigenvalues. The absence of any more interesting behavior indicates that the linearized U-U equation may be incapable of describing the Bose gas very close to the phase transition.

In conclusion, the Uehling-Uhlenbeck equation performs best as a refinement to the Boltzmann equation at the level of quantum corrections. It cannot accurately describe new processes that occur within quantum regime such as BoseEinstein condensation or superfluidity. However, we find that initial distributions which are close to the quantum regime
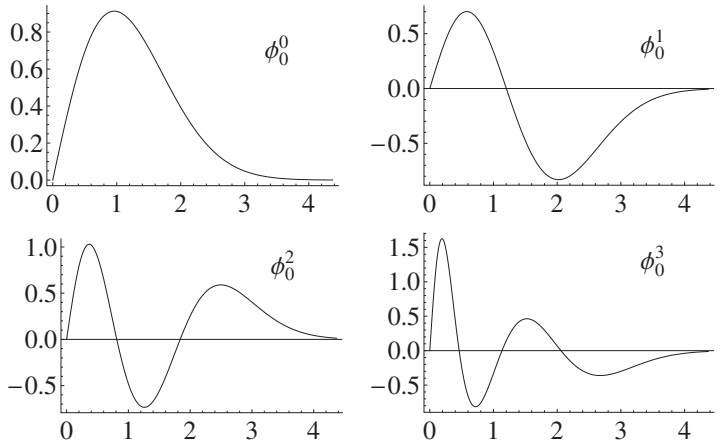

FIG. 6. A plot of $\phi_{l}^{n}$ versus $c$ showing the first few eigenvectors when $l=0$ and $z=0.1$.
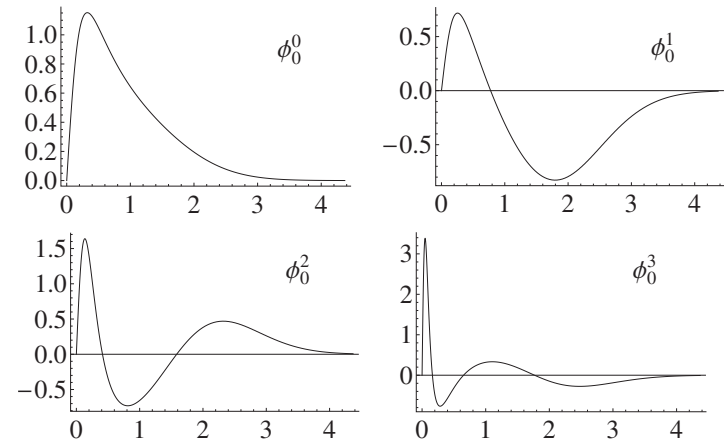

FIG. 7. A plot of $\phi_{l}^{n}$ versus $c$ showing the first few eigenvectors when $l=0$ and $z=0.9$.

will relax to equilibrium at a much faster rate than if they were treated classically. We also conclude that spectrum of the linearized Uehling-Uhlenbeck equation predicts that distributions near equilibrium will relax exponentially toward a Bose-Einstein equilibrium distribution.

\section{ACKNOWLEDGMENT}

The authors wish to thank the Robert A. Welch Foundation (Grant No. F-1051) for support of this work.

\section{APPENDIX A: EVALUATION OF THE FUNCTIONS $Q$ and $S$}

In this appendix we outline the derivation of the analytical forms for the functions $Q$ and $S$ given in Eqs. (15) and (16). We also give the classical limit of these equations and show that it agrees with the result from the Boltzmann equation for a gas of hard spheres.

\section{Evaluation of the function $Q$}

Here, we outline the steps to arrive at Eq. (15) from Eq. (12). We begin by defining a more symmetric set of integration variables, $\mathbf{p}=\frac{1}{2}\left(\mathbf{c}_{3}+\mathbf{c}_{4}\right)$ and $\mathbf{q}=\mathbf{c}_{3}-\mathbf{c}_{4}$. In terms of these, Eq. (12) becomes

$$
\begin{aligned}
Q\left(\mathbf{c}_{1}, \mathbf{c}_{2}\right)= & \frac{2}{\pi} \int d \mathbf{p} d \mathbf{q} \delta^{3}\left(\mathbf{c}_{1}+\mathbf{c}_{2}-2 \mathbf{p}\right) \delta\left(c_{1}^{2}+c_{2}^{2}-2 p^{2}-\frac{q^{2}}{2}\right) \\
& \times\left(1+\frac{z}{e^{(\mathbf{p}+\mathbf{q} / 2)^{2}}-z}\right)\left(1+\frac{z}{e^{(\mathbf{p}-\mathbf{q} / 2)^{2}}-z}\right) \cdot \quad(\mathrm{A} 1
\end{aligned}
$$

The $\delta$ functions cancel several of the integrals, leaving us with

$$
\begin{aligned}
Q\left(\mathbf{c}_{1}, \mathbf{c}_{2}\right)= & \frac{q}{2} \int_{-1}^{1} d \alpha\left[1+\frac{z}{e^{p^{2}+q^{2} / 4} e^{p q \alpha}-z}+\frac{z}{e^{p^{2}+q^{2} / 4} e^{-p q \alpha}-z}\right. \\
& \left.+\frac{z^{2}}{\left(e^{p^{2}+q^{2} / 4} e^{p q \alpha}-z\right)\left(e^{p^{2}+q^{2} / 4} e^{-p q \alpha}-z\right)}\right], \quad \text { (A2) }
\end{aligned}
$$

where $p=\frac{1}{2}\left|\mathbf{c}_{1}+\mathbf{c}_{2}\right|, q=\left|\mathbf{c}_{1}-\mathbf{c}_{2}\right|$, and $\alpha=\hat{\mathbf{c}}_{1} \cdot \hat{\mathbf{c}}_{2}$. The final integration over $\alpha$ results in 


$$
Q\left(\mathbf{c}_{1}, \mathbf{c}_{2}\right)=\frac{e^{2 p^{2}+q^{2} / 2}}{e^{2 p^{2}+q^{2} / 2}-z^{2}}\left[\frac{1}{p} \ln \left(\frac{e^{p^{2}+p q+q^{2} / 4}-z}{e^{p^{2}-p q+q^{2} / 4}-z}\right)-q\right] .
$$

Rewriting this in terms of $\mathbf{c}_{1}$ and $\mathbf{c}_{2}$ gives us Eq. (15). The function $Q$ is manifestly symmetric in an interchange of $\mathbf{c}_{1}$ and $\mathbf{c}_{2}$. In the classical limit when $z \rightarrow 0$, we can just replace $z$ with zero in the expression for $Q$. The result simplifies to the well-known $[8,15]$ classical expression for hard spheres,

$$
Q_{\mathrm{CL}}\left(\mathbf{c}_{1}, \mathbf{c}_{2}\right)=\left|\mathbf{c}_{1}-\mathbf{c}_{2}\right| \text {. }
$$

\section{Evaluation of the function $S$}

For evaluation of the function $S$ from Eq. (13), we again define the integration variables $\mathbf{p}$ and $\mathbf{q}$ by the same formulas as in the previous section. In terms of these, Eq. (13) becomes

$$
\begin{aligned}
S\left(\mathbf{c}_{1}, \mathbf{c}_{2}\right)= & \frac{2}{\pi z} e^{c_{2}^{2}} \int d \mathbf{p} d \mathbf{q} \delta^{3}\left(\mathbf{c}_{1}-\mathbf{c}_{2}+\mathbf{q}\right) \\
& \times \delta\left(c_{1}^{2}-c_{2}^{2}+2 \mathbf{p} \cdot \mathbf{q}\right) \frac{z}{e^{[\mathbf{p}+(1 / 2) \mathbf{q}]^{2}}-z} \\
& \times\left(1+\frac{z}{e^{[\mathbf{p}-(1 / 2) \mathbf{q}]^{2}}-z}\right) .
\end{aligned}
$$

It is easiest to evaluate the $\mathbf{q}$ integration first and then evaluate the $\mathbf{p}$ integration in cylindrical coordinates with the $z$ axis oriented in the direction of $\mathbf{q}$. After doing this, we obtain

$$
\begin{aligned}
S\left(\mathbf{c}_{1}, \mathbf{c}_{2}\right)= & \frac{2}{q z} e^{c_{2}^{2}} \int_{0}^{\infty} p_{\rho} d p_{\rho} \\
& \times\left(1+\frac{z}{e^{p_{\rho}^{2}+p_{z}^{2}+q^{2} / 4-p_{z} q}-z}\right) \frac{z}{e^{p_{\rho}^{2}+p_{z}^{2}+q^{2} / 4+p_{z} q}-z},
\end{aligned}
$$

where $p_{z}=\left(c_{2}^{2}-c_{1}^{2}\right) / 2 q$ and $q=\left|\mathbf{c}_{1}-\mathbf{c}_{2}\right|$.

The remaining integral over $p_{\rho}$ can be evaluated by defining $u=e^{p_{\rho}^{2}}$. Rewriting this result in terms of $\mathbf{c}_{1}$ and $\mathbf{c}_{2}$ gives us Eq. (16). This equation is also symmetric under interchange of $\mathbf{c}_{1}$ and $\mathbf{c}_{2}$. In the $z \rightarrow 0$ limit we cannot simply replace $z$ in Eq. (16) with zero due to the appearance of $z$ in the denominator. A quick inspection reveals that the numerator also goes to zero as $z$ approaches zero. Expanding the logarithm in Eq. (16) in powers of $z$, we find that

$$
\begin{aligned}
S\left(\mathbf{c}_{1}, \mathbf{c}_{2}\right)= & \frac{1}{z\left|\mathbf{c}_{1}-\mathbf{c}_{2}\right|\left(e^{-c_{1}^{2}}-e^{-c_{2}^{2}}\right)} \\
& \times\left[e^{\left|\mathbf{c}_{1} \times \mathbf{c}_{2}\right|^{2} /\left(\mathbf{c}_{1}-\mathbf{c}_{2}\right)^{2}}\left(e^{-c_{1}^{2}}-e^{-c_{2}^{2}}\right) z+O\left(z^{2}\right)\right],
\end{aligned}
$$

which is indeed equal to the classical result for hard spheres $[8,15]$,

$$
S_{\mathrm{CL}}\left(\mathbf{c}_{1}, \mathbf{c}_{2}\right)=\frac{1}{\left|\mathbf{c}_{1}-\mathbf{c}_{2}\right|} e^{\left|\mathbf{c}_{1} \times \mathbf{c}_{2}\right|^{2 / /\left(\mathbf{c}_{1}-\mathbf{c}_{2}\right)^{2}}}
$$

\section{APPENDIX B: THE FORM OF THE SPECTRUM AND THE START OF THE CONTINUUM}

In this appendix, we outline the steps necessary to obtain Eq. (33). Numerical computation of the spectrum of discrete eigenvalues for the U-U equation indicates that it takes the same form as it does for the classical Boltzmann equation for hard spheres. A plot of $K^{l}\left(c_{1}, c_{2}\right)$ shows that it is continuous and probably completely continuous (although no proof currently exists). The spectrum of the U-U equation contains two parts: a bounded region of discrete eigenvalues and an unbounded region of continuous eigenvalues. The discrete eigenvalues accumulate at the boundary between the discrete and continuum regions. This boundary point is denoted by $\lambda_{L}(z)$ and is given by the expression that multiplies $\gamma g_{l, m}\left(c_{1}, t\right)$ in Eq. (21), evaluated at $c_{1}=0$ [10].

We begin the calculation with the definition

$$
\lambda_{L}(z)=M(0) .
$$

After substituting in from Eqs. (2), (15), (19), and (20) we get

$$
\begin{aligned}
\lambda_{L}(z)= & \frac{1-z}{z} \int_{0}^{\infty} d c_{2} c_{2}^{2} \frac{z}{e^{c_{2}^{2}}-z} \int_{-1}^{1} d(\cos \theta) \\
& \times P_{0}(\cos \theta) \frac{e^{c_{2}^{2}}}{e^{c_{2}^{2}}-z^{2}}\left[\frac{2}{c_{2}} \ln \left(\frac{e^{c_{2}^{2}}-z}{1-z}\right)-c_{2}\right] .
\end{aligned}
$$

The integral over $\theta$ is trivial and the integral over $c_{2}$ can be evaluated analytically in terms of the polylogarithm function $\mathrm{Li}_{2}(z)$. The result is

$$
\lambda_{L}(z)=\frac{1}{z}\left[-2 \mathrm{Li}_{2}(-z)-\mathrm{Li}_{2}(z)+\mathrm{Li}_{2}\left(z^{2}\right)\right] .
$$

Using an identity which can be easily proved from the definition $\mathrm{Li}_{2}\left(z^{2}\right)=2 \mathrm{Li}_{2}(z)+2 \mathrm{Li}_{2}(-z)$, this simplifies to

$$
\lambda_{L}(z)=\frac{\operatorname{Li}_{2}(z)}{z} .
$$

Figures 4 and 5 show a plot of $\lambda_{L}(z)$ along with several eigenvalues. 
[1] E. A. Uehling and G. E. Uhlenbeck, Phys. Rev. 43, 552 (1933).

[2] S. Kikuchi and L. Nordheim, Z. Phys. 60, 652 (1930).

[3] C. J. Pethick and H. Smith, Bose-Einstein Condensation in Dilute Gases (Cambridge University Press, Cambridge, England, 2002), pp. 142-145.

[4] M. J. Bijlsma, E. Zaremba, and H. T. C. Stoof, Phys. Rev. A 62, 063609 (2000).

[5] M. S. Child, Molecular Collision Theory (Academic Press, New York, 1974), pp. 21-39.

[6] K. B. Davis, M. O. Mewes, M. R. Andrews, N. J. van Druten, D. S. Durfee, D. M. Kurn, and W. Ketterle, Phys. Rev. Lett. 75, 3969 (1995).

[7] N. R. Newbury, C. J. Myatt, and C. E. Wieman, Phys. Rev. A 51, R2680 (1995).
[8] E. D. Gust and L. E. Reichl, Phys. Rev. E 79, 031202 (2009).

[9] S. Chapman and T. G. Cowling, Mathematical Theory of NonUniform Gases (Cambridge University Press, Cambridge, England, 1970).

[10] I. Kuščer and M. M. R. Williams, Phys. Fluids 10, 1922 (1967).

[11] B. Shizgal, Can. J. Phys. 62, 97 (1984).

[12] J. Kondo, Integral Equations (Clarendon Press, Oxford, 1991), Chap. 6.

[13] P. L. Bhatnagar, E. P. Gross, and M. Krook, Phys. Rev. 94, 511 (1954).

[14] D. Kahaner, C. Moler, and S. Nash, Numerical Methods and Software (Prentice-Hall, Englewood Cliffs, 1989), pp. 153157.

[15] O. O. Jenssen, Phys. Norv. 6, 179 (1972). 